\title{
SizelTALY - The Actual Italian Measurement Survey
}

\author{
Peter V. STAMPFLI*a, Anke RISSIEK ${ }^{\mathrm{b}}$, Rainer TRIEB ${ }^{\mathrm{b}}$, Andreas Seidl $^{\mathrm{b}}$ \\ ${ }^{a}$ Sistemi Assyst s.r.l., Lainate (MI), Italy;

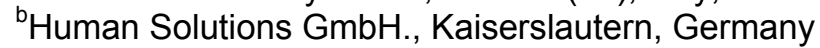

\begin{abstract}
In August 2012 a representative anthropometrical measurement study in Italy, called SizeITALY, has been started. Within a period of 12 month 6,000 women, men and children will be measured using 3D-body scanners. The objective of the survey is to analyze and document the changes in more than 80 body measurements and 3D-body shapes in comparison to ancient studies. The apparel industry will be provided with updated body measurement statistics (ISO 8559-compliant). Additionally actual size charts will be developed and market shares will be calculated. 3D-body shapes will submit the apparel industry in the integration of 3D virtual product development processes into their daily business. Based on the results also updated body measurement statistics (ISO 7250-compliant) are developed and an updated 3D model for the CAD man model RAMSIS will be developed for the automotive industry and other ergonomic oriented industries. The complete results will be published in a web portal called iSize which already consists of survey data from 8 other countries worldwide.

This paper will present the objectives of the participating companies, the conceptual design of the study and the scientific and technical challenges. It will enlighten the project under the framework of global comparison of survey results and their benefits for the apparel industry and ergonomics as well as for the implementation of 3D-development facilities in the apparel industry.
\end{abstract}

Keywords: 3d body scanning, measurement survey, sampling strategy, comparison of international survey data

\section{Introduction}

Anthropometric design is one of the most important disciplines within the area of ergonomic approaches. With the correct anthropometry layout, products and working tools can be used safely, comfortably and in a healthy manner. This is why many anthropometric studies were initiated in the last century - and in the second half of that century anthropometric surveys were organized on a global scale [6]. Most of these were initiated by the logistic departments of national armies [5]. Organized by the German Fashion Association, the last survey in Germany for women was carried out in 1992/94 however, a survey for the reunified Germany or a survey for children had not been carried out.

The beginning of the 21st century saw a new era of anthropometric surveys. CAESAR (Civilian American and European Surface Anthropometry Resource) was the first survey which used a 3D body scanner to acquire 3D scans of body surfaces, as well as demographics data and traditional measurements [17]. In 1999 and 2000, 11,000 subjects in Great Britain were scanned and manually measured (SizeUK). The "next step of evolution" was made in France in 2003-2005. More than 11,000 subjects were scanned using a new technology, so parallel manual measurements were no longer used. The Swedish survey of 2004/2005 scanned more than 4,000 subjects, using the same technology and concept as the French survey. 2007 saw the initiation of several body scanning surveys in other countries like Spain and China.

A new survey was also started in Germany in 2008/2009, called SizeGERMANY, where more than 12,000 women, men and children where measured at more than 30 different scanning locations. Especially the German apparel industry can be characterized by its great success in the export sector. This is why the need of global anthropometric data is obvious additionally to local market knowledge. Although regularly surveys are conducted in the fast growing Asian markets, as China or Japan, there is a lack of information about the Southern European and even much more about the Eastern European markets $[13,14]$.

\footnotetext{
* peter.stampfli@assyst.de; +39 0293769 223; http://www.assyst-intl.com
} 
Within Southern Europe an anthropometric study about the female population in Spain was promoted $2007 / 2008$ by the Ministry of Health supported by the main Spanish companies from the apparel industry. 10,414 women between 18 and 65 years were measured by the Institute of Biomechanics of Valencia with 3D body scanner technology. The medium body height of the women in Spain is 162.3 $\mathrm{cm}$, which is $4.5 \mathrm{~cm}$ less than the medium body height in Germany $(166.73 \mathrm{~cm})$. This indicates that there are significant differences in body measurements between the different European region and countries and underlines the necessity for actual measurement data from Italy [1].

\section{A short history about size surveys in Italy}

Already 3 previous studies have been conducted to measure the Italian population: Between July 1990 and September 1991 the anthropometric study "L'Italia si misura", carried out by Ente Nazionale Italiano di UNIficazione, measured 4021 male and female subjects between 18 and 65 years. The survey used traditional anthropometric measuring methods and differentiated between Center-North, South and the area around Rome. Within this survey significant differences of 3-4 cm in body height between Center-North and South Italy occurred for both, male and female. The results also showed significant differences of 6-7 cm in body height between the younger and the elderly age groups for both, male and female, too. The results were published in the he ISO 7250-2 for the anthropometry apparel measurements have not been analyzed $[7,11]$.

The Civilian American and European Surface Anthropometry Resource (CAESAR) project was a survey of the civilian populations of three countries representing the North Atlantic Treaty Organization (NATO) countries: the United States of America (USA), The Netherlands, and Italy (Robinette et al. 1999, Robinette 2000). The survey was carried out by the U.S. Air Force, with the help of the contractor Sytronics Inc., the Netherlands Organization for Applied Scientific Research (TNO), the subcontractor D'Appolonia in Italy and a consortium of companies under the umbrella of the Society of Automotive Engineers (SAE). CAESAR was the first survey using 3D-bodyscanning technology to update and augment body measurement data bases. Within Italy 410 males and 386 females have been measured in South Italy. The 3D body scans were carried out in 3 different measurement positions with reference to the CAESAR measurement set $[8,17]$. CAESAR showed a difference in body height for male $(>4 \mathrm{~cm})$ and female $(>3 \mathrm{~cm})$. The scientists found a significant increase of weight from younger to elderly people $(5.5 \mathrm{~kg}$ for male and $>8 \mathrm{~kg}$ for female. Regional analysis is not available because of the one measurement site in the south of Italy; body measurements according to ISO 8559 for the apparel industry have not been analyzed.

The latest anthropometric study in Italy was the OEC-Cardiovascular Epidemiologic Observatory survey between 1998 and 2002. Its objectives were to identify the risk factors as well as the frequency of risk conditions and prevalence of cardiovascular diseases within the Italian population. The sampled population was a representative mixture of 4.908 men and 4.804 women between 35-74 years in 4 different regions (North East, North West, Centre and South and Islands) within 51 medical centers all over Italy. The OEC found significant differences in waist and hip circumferences in the 4 regions, whereas the maximum differences were carried out between Centre and South and showed inhomogeneous figure type characteristics within male and female population [12]. Table 1 presents the results for male and female in the different regions in ITALY.

Table 1. Results from OEC Cardiovascular Epidemiologic Observatory survey.

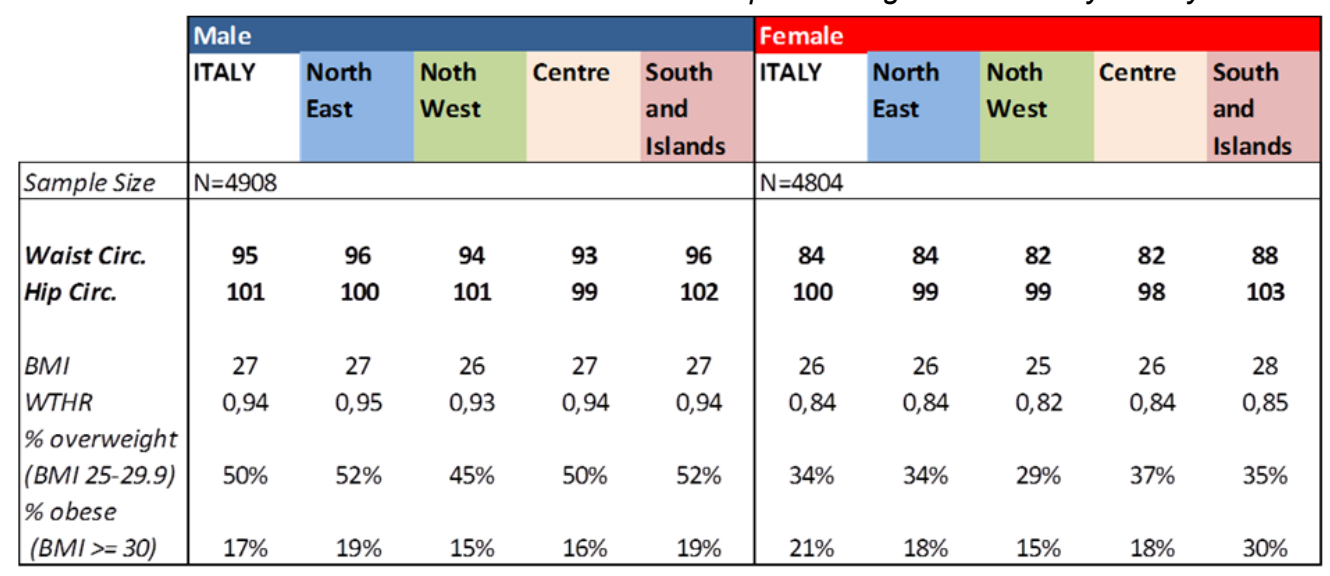


The results from the OEC Cardiovascular Epidemiologic Observatory survey specifically showed the largest difference between the regions North and South whereas the difference between North-East, North-West and Centre is negligible [2].

\section{Conceptual Design of SizelTALY}

\subsection{Partnership concept}

Italy is the $4^{\text {th }}$ largest population in Europe and has an impressive tradition in the fashion industry. In the year 2007 there were 58,000 companies in the fashion industry with 513,000 employees. Their yearly revenues were 54 Mio. Euros in 2007 with an export rate of more than 50 percent. The fashion business is therefore an important industry sector for the Italy $[15,16]$.

Italy is the most active fashion industry in the world, in terms of quantity, second only to China. As a lot of the companies engaged in the Italian fashion business are smaller or medium-size companies, the partnership model has to offer economically interesting opportunities also for smaller enterprises. This is the reason why the participation model depends on the yearly turnover and regards the area of results (women, men and children in all combinations).

Partners in the project receive the following results:

- Up to date body measurements differentiated for the apparel and the automotive industry

- Up to date size tables for women, men and children (corresponding to subscription range)

- Up to date market shares for women, men and children (corresponding to subscription range)

- Results of the socio-demographic survey

- Integrated results in the iSize portal

Actually SizelTALY has partners from all branches in the fashion business. Participation is still open until the end of 2012. An important part of the partnership concept is an already installed steering committee that is especially responsible for technical accompaniment of SizelTALY as well as for the long term usage of SizelTALY results. YAMAMAY (http://www.yamamay.com/it), the famous Italian lingerie company, is one of the companies in the steering committee and provides the complete underwear for the survey that is given as a gift to the measured persons after scanning.

\subsection{Sampling strategy}

Surveys are nowadays often financed privately by the industry that will in the future use the results. This leads to the requirement of minimum subjects to reduce measurement costs and at the same time to measure as many subjects that are needed to carry out representative results. Sampling strategy is therefore often a necessity for economic success of a survey project.

The primary requirement on anthropometrical surveys is the representation of the entire population. In most of the survey carried out manually, two tenths of one percent of the population was regarded as being representative. Within SizeGERMANY a new approach was used: During the survey the current database was constantly aligned with the statistically necessary data. Each person stored in the database was classified by gender, ager and regional distribution. A dynamic monitoring of the measured sample with the necessary data range lead to a minimum number of measured subjects.

The results and the experience with this new methodology of this previous study lead to the following conclusions for the conceptual design of SizelTALY:

1. Body height as a length dimension and weight as width measurements are both influenced by age (body height decreases nearly linear with an increasing age). A detailed age classification is necessary to carry out those age-specific changes.

2. A regional diversification is needed to take into account the measurement differences between the different regions in Italy. As the most significant differences in body measurements occur between North/Center Italy and South Italy a minimum of 2 regional samples has to be performed.

3. Due to the comparability to the results of other surveys the partner group decided on 6 age groups for adults between 18 and 75 years and 3 age groups for children between 6 and 17 years (same age classification has already been used in France and Germany). 
Based on accuracy requirements of the industrial partners (10 $\mathrm{mm}$ for body height and 955 confidence) the sample size has to be minimal 165 persons per group.

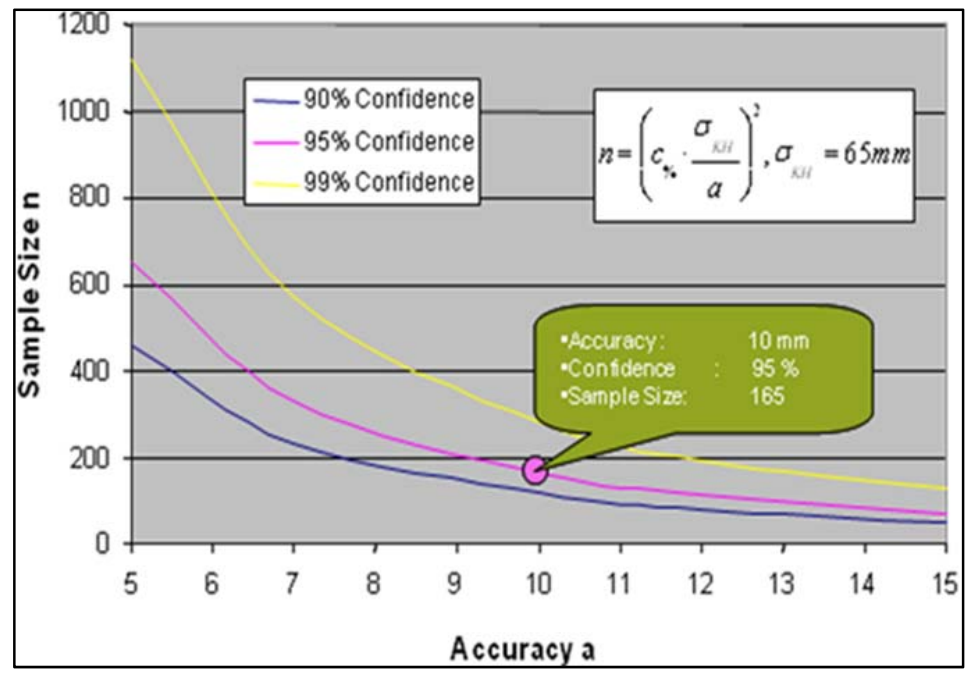

Fig. 1. Relation of sample size and required accuracy.

These requirements as a whole lead to a minimum sample size of 6,000 persons (number of subjects $=$ groups size ${ }^{*}$ number of age groups ${ }^{*}$ number of regions; $165{ }^{*} 9{ }^{*} 2=6,000$ ).

\subsection{Selection of measurements sites}

The official start of SizeITALY was August 2012 with the first measurement site at the Sistemi office at Lainate (MI). This measurement site was especially used for training of the personnel. After that the scanner moved to Ostia Beach (region North-Center of Italy) where within August about 500 scans have been performed. Due to the positive experience with measurement sites at beaches where people have already put out their clothes the next measurement site is Naples, Palmarey (region South Italy).

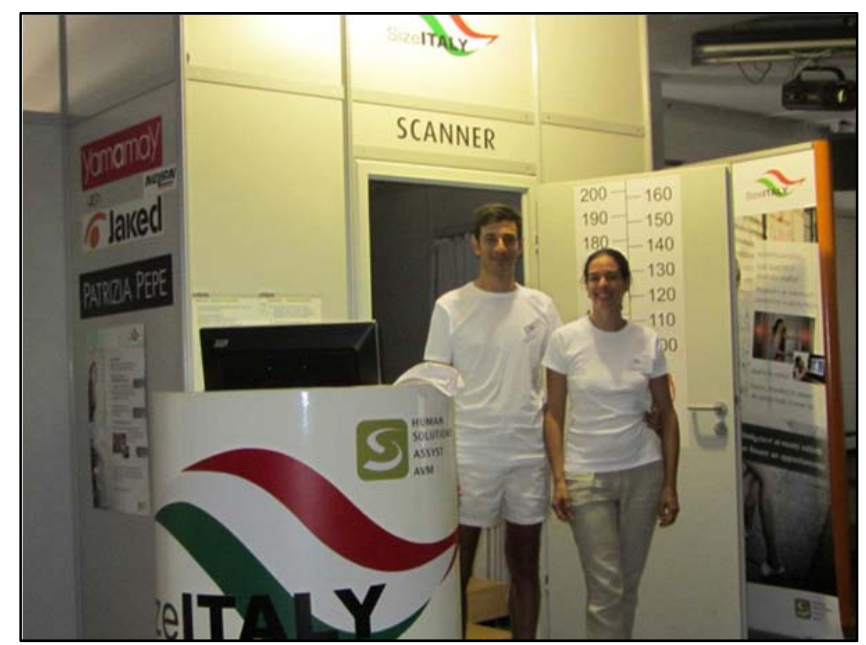

Fig. 2: Measurement site at Ostia Beach, Kursaal.

As it has been already performed successfully in SizeGERMANY, too, participating companies can provide locations for measurement sites. The experience shows that the marketing advantage for the companies is enormous. In SizelTALY the first company that provides a measurement location is ACON at San Martino, Verona.

After the first 2 measurement sites the current status of scanned persons is shown in Figure 4. According to the first measurement sites in the North of Italy, the number of scanned persons from the north is significantly higher than from the South. 


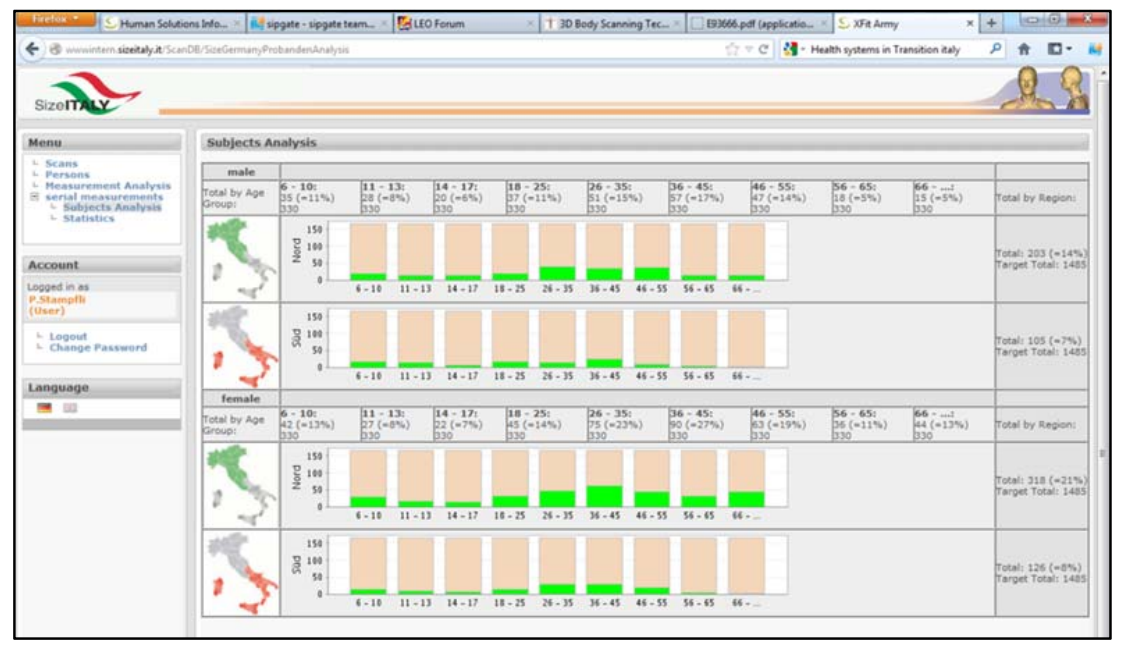

Fig. 3. Subject Analysis for sampling.

As the current status is analyzed on a daily basis the next measurement sites can be selected with respect to those population groups and age classes that are needed. A current quality control of scan data on a daily basis guarantees excellent results.

\subsection{D Body Scanning}

Goal of the survey is to scan people in 4 different measurement positions (3 different standing positions and one seated position) at 12-15 measurement sites within a measuring period of 12-15 months.

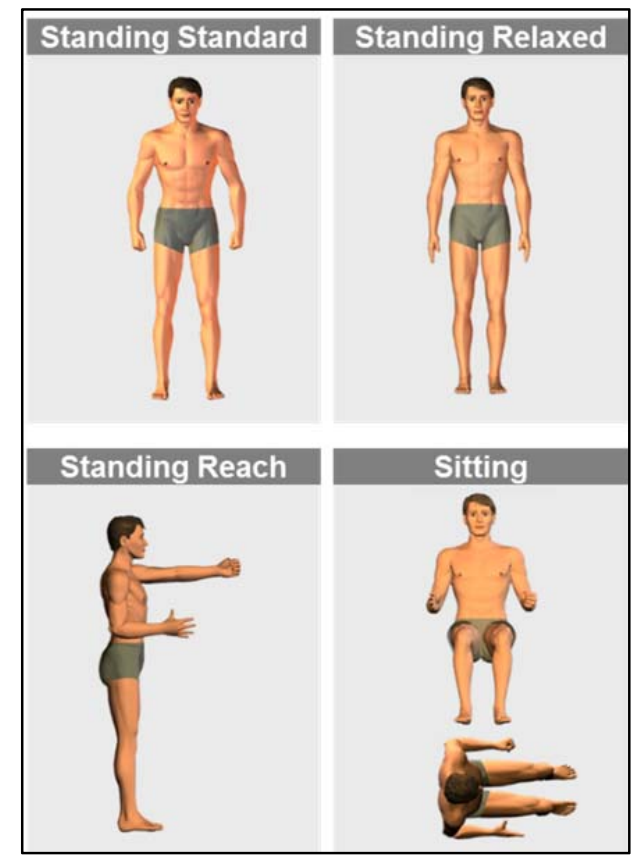

Fig. 4. Scanning postures for SizeITALY.

\subsection{Socio-demographic Questionnaire}

The socio-demographic questionnaire used within SizelTALY is predicated on the questionnaire used within the SiteGERMANY survey. This will allow comparisons between the results of SizeGERMANY and SizelTALY as well as cross-country analysis of the combined markets within iSize. 
The questionnaire comprehends different category groups starting with the socio-demographic characteristics as gender, age group, place of residents and the level of education. The second category group consists of apparel related questions as for example the interest in fashion, buying habits or actual fit problems. The third and the forth category are for the automotive as well as for the motorcycle industry and consists of questions about the actually driven cars and motorbikes.

\subsection{Body measurements}

Body measurements have to be determined according to technical market standards to achieve the highest degree of comparability and combinability.

The apparel measurement set in SizelTALY consists of 44 measurements widely compliant with the ISO 8559 international standard for garment design [4]. These measurements are taken completely from a standard standing posture of the measured subjects.

\begin{tabular}{|c|c|c|c|}
\hline \multicolumn{2}{|c|}{ Primary measurements } & \multicolumn{2}{|c|}{ Secondary measurements } \\
\hline 1 & Height & 26 & Upper Arm Length \\
\hline 2 & Bust/Chest Girth & 27 & Upper Arm Girth \\
\hline 3 & Hip Girth & 28 & Elbow Girth \\
\hline 4 & Underbust Girth & 29 & Middle Forearm Girth \\
\hline 5 & Waist Girth & 30 & Wrist Girth \\
\hline 6 & Waist Height & 31 & Arm Length - 7th Cervical Vertebra - Acromion - \\
\hline 7 & Outside Leg Length & & Elbow - Wirst \\
\hline 8 & Inside Leg Length & 32 & Middle Hip (replacement for circumference $8 \mathrm{~cm}$ \\
\hline \multicolumn{2}{|c|}{ Secondary measurements } & & \\
\hline 9 & Neck Girth & 33 & Maximum Waist Girth \\
\hline 10 & Neck - Base Girth & 34 & Thigh Girth \\
\hline 11 & $\begin{array}{l}\text { Neck-Base Girth } \\
\text { Head Girth }\end{array}$ & 35 & Knee Girth \\
\hline 12 & $\begin{array}{l}\text { Head Girth } \\
\text { Neck Base Diameter (front view }\end{array}$ & 36 & Upper Knee Girth \\
\hline 13 & Neck Base Diameter (front view & 37 & Lower Knee Girth \\
\hline 10 & Shoulder Length & 38 & Calf Girth \\
\hline 14 & Shoulder Slope & 39 & Minimum Leg Girth \\
\hline 15 & Back Width & 40 & Cervical Heigth \\
\hline 16 & Back Waist Length - (7th cervical vertebra to waist) & 41 & Cervical to Knee Hollow \\
\hline 17 & Front Waist Length & 42 & Knee Height \\
\hline 18 & Neck Shoulder Point to Breast Point & 43 & Shine bone (cnemis) Height \\
\hline 19 & Bust Width & 44 & Weight \\
\hline 20 & Bust/Chest Height & & \\
\hline 21 & Trunck Length & & \\
\hline 22 & Waist to Hip & & \\
\hline 23 & Total Crotch Length & & \\
\hline 24 & Crotch Length Front & & \\
\hline 25 & Arm Length & & \\
\hline
\end{tabular}

Fig. 5.Body Measurements Apparel for SizelTALY.

The Ergonomic measurement set consists of 37 measurements compliant with the ISO 7250 international standard for technological design [3]. These measurements are taken partially from the standing, the sitting and the functional posture of the measured subjects.

Additionally, a set of commonly used indices is provided by SizelTALY. This includes BMI (Body Mass Index), WTHR (Waist-to-Hip-Ratio) and Bust-to-Underbust-Difference which is needed for figure type classification during design and optimization of size tables. 


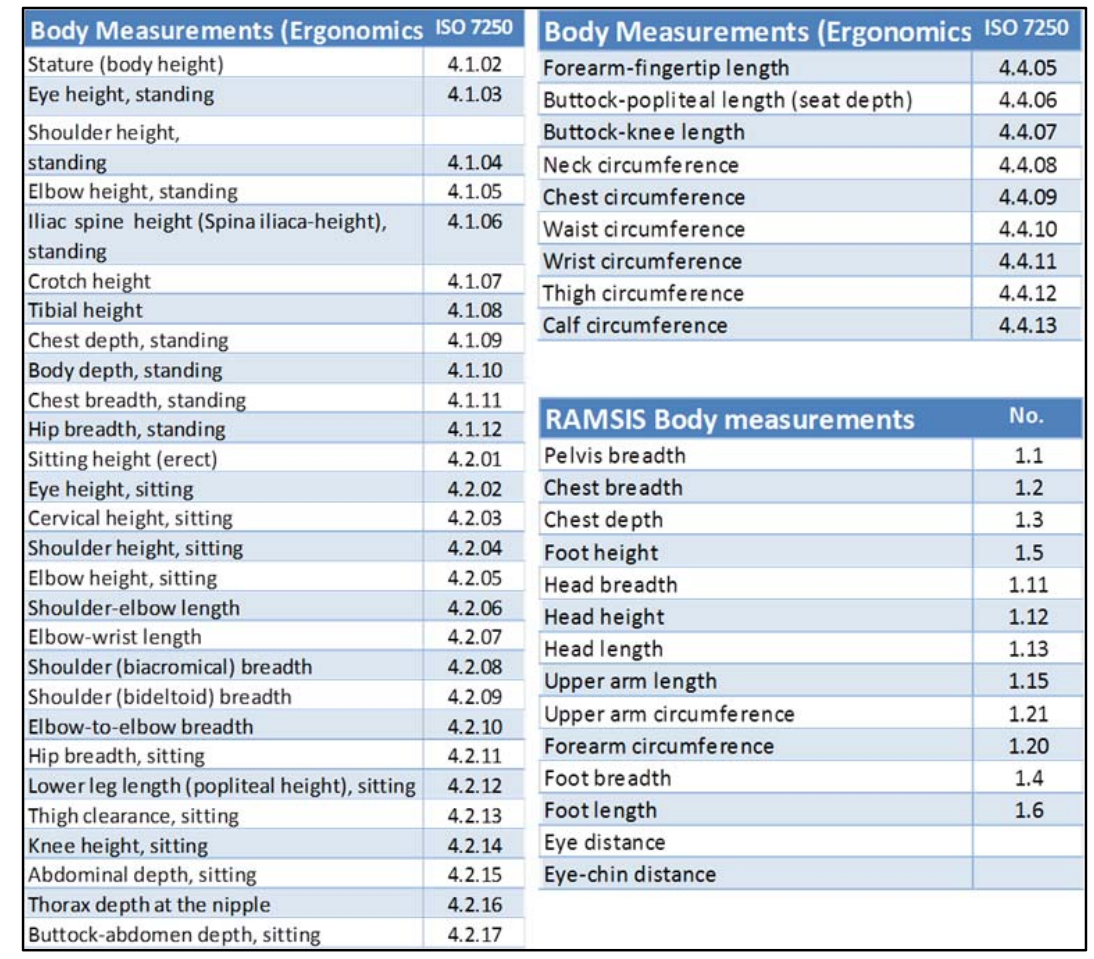

Fig. 6.Body Measurements Ergonomics for SizelTALY.

\subsection{Actual state of activities}

The actual planning for SizelTALY divides the complete survey in 4 different phases with several key aspects of activity. The planning for the complete project has been finished and the data collection has already started and will last until the end of next year. The analysis phase is partly performed in parallel to the measurement phase, starting with quality control of the scans and landmark checks. The analysis phase will be completed with the integration of the data into the iSize portal and with the final presentation of the results to the project partners. In parallel the acquisition of new partners for SizelTALY is handled. Figure 6 shows an overview of all project steps and the complete time table.

\section{Integration of results into the iSize portal}

All results and analyses will be available in international data portal iSize and allow a direct comparability and possibility to combine with the results of SizeGERMANY or with the other available data from France, the Netherlands, Switzerland, China, Japan, Korea and the USA. This will help to determine significant differences of measurements between different markets and support the decisions of product designers about different sizing systems or basic ergonomic design models [9].

As for example the Fashion industry is internationally oriented with a high percentage of export activities the challenge is to sell the collections worldwide with a perfect fit for each market. This is nearly impossible because the development costs are so high that a company cannot generate an individual fit for each market. From the economic side fit has therefore always to be the best compromise.

If apparel companies want to deliver their products to foreign markets they have to decide either to live with a compromise fit or to face the enormous costs for the development of individual collection for each single market [10].

This decision process can be additionally supported by medium 3D body shapes representing the typical customers in a certain size of a size table. These 3D-shapes will be provided for SizelTALY, too. 

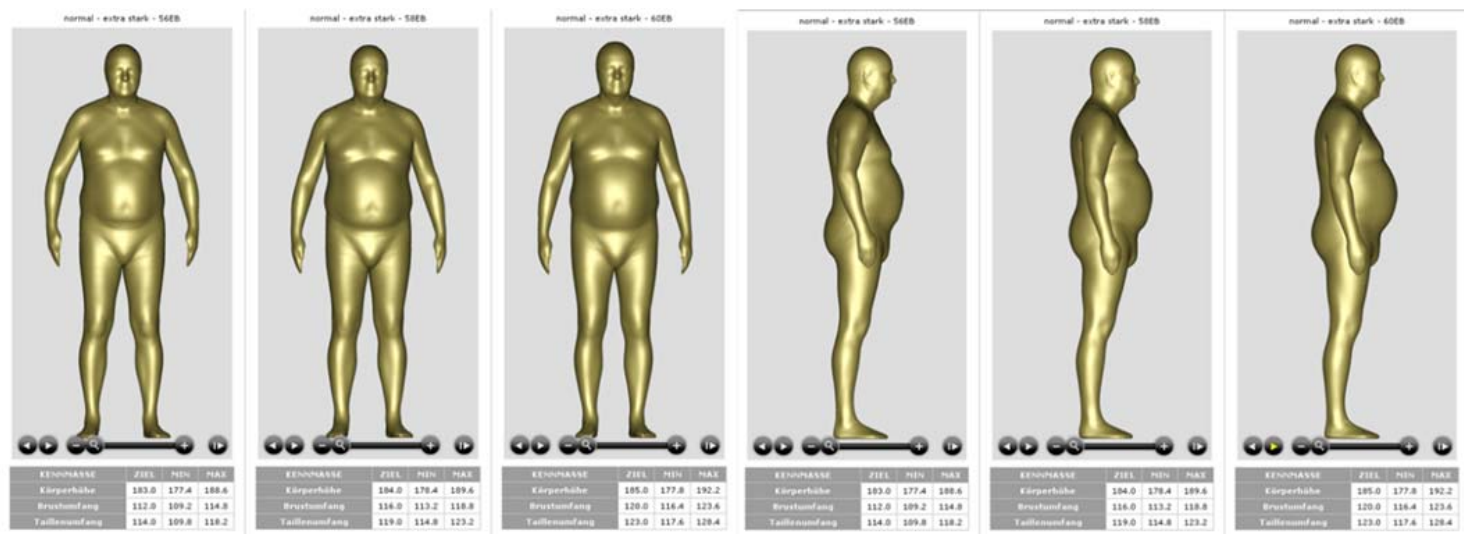

Fig. 7.Generation of 3D body shapes in the international data portal iSize.

\section{Outlook}

The results from the Italian survey SizeITALY will be available in December 2013 within the iSize portal. The participants of the survey will then have a detailed overview on the body measurements and 3D-shapes of the Italian population. In a first step they will then have the chance optimize their fit for the local Italian market.

Furthermore they will profit from the cross-country comparisons iSize allows and with this knowledge they have the opportunity to achieve a higher competitiveness on international markets in the future, too.

\section{References}

1. Alemany, S., Gonzáles, J. C., Nácher, B., Soriano, C., Arnáiz, C., Heras, Á. Anthropometric Survey of the Spanish Female Population Aimed at the Apparel Industry. International Conference on 3D Body Scanning Technologies, Lugano, October 2012, p. 303-311.

2. Information about OEC Cardiovascular Epidemiologic Observatory survey: http://www.cuore.iss.it/eng/factors/obesity.asp

3. ISO 7250:1996: "Basic human body measurements for technological design".

4. ISO 8559:1989: "Garment construction and anthropometric surveys -- Body dimensions".

5. Jürgens, H. W., Aune, I. A., Pieper, U. (1989). Internationaler anthropometrischer Datenatlas. Schriftenreihe der Bundesanstalt für Arbeitsschutz. Fb 587.

6. Jürgens, H. W. (1992). Anwendung anthropometrischer Daten. Direkte und indirekte Anwendung. In Schmidtke, H. Handbuch der Ergonomie (A - 3.3.1). Hanser Verlag.

7. Pierlorenzi, G. (2012): L'Italia si misura vol.I, Narcissus Self Publishing (19. Januar 2012).

8. Robinette, K. M., Daanen, H. A. M., Zehner, G. F. (2004). Three Dimensional Anthropometry. In Delleman, N.J., Haslegrave, C.M., Chaffin, D.B. Working Postures and Movements (pp 29-49). CRC Press LLC.

9. Rissiek, A., Trieb, R.: iSize - Implementation of international anthropometric survey results for worldwide sizing and fit optimization in the apparel industry. International Conference on 3D Body Scanning Technologies, Lugano, October 2010, p. 265-277.

10. Rissiek, A.: "Morgen verkaufen wir auch in Asien! Strategien und Lösungen zur Optimierung von Marktpotenzial und Passform für internationale Märkte“. Fashion Forum, Munich, 2009.

11. Salis N. A. (2002): Anthropometric survey of Italian Population L'Italia si Misura: A Decade of Research. In: De Waard, Brookhuis, Mooral, Toffetti. Human Factors in Transportation, Communication, Health and the Workplace. (pp. 409-420). ISBN: 90-423-0206-2.

12. Scalzo, A. L.; Donatini, A., Orzella, L., Cicchetti, A., Profili, S., Maresso, A. (2009): ITALY - Health System Review. Health systems in Transition, Vol. 11, No. 6, pp.3.

13. Seidl, A., Trieb, R., Wirsching, H. J.: SizeGERMANY - die neue Deutsche Reihenmessung - Konzeption, Durchführung und erste Ergebnisse. 54. Frühjahrskongress der Gesellschaft für Arbeitswissenschaft, 2008.

14. Seidl, A., Trieb, R., Wirsching, H. J.: SizeGERMANY - the new German Anthropometric Survey. Conceptual Design, Implementation and Results. IEA 2009, Peking.

15. Statistics Italian Fashion industry: http://www.sistemamodaitalia.com/Pri/Hom.asp?gsAppLanCur=EN\&gsPagTyp=2\&gsMnuNav=01M:100,01L: 3,01C:2,02M:103,02L:2,02C:2

16. Tronconi, M.: The Italian Fashion Industry: the secrets of Made in Italy. LIUC - Strategic issues of Made in Italy. Castelanza, 26.02.2012, Sheet 22.

17. United Sates Air Force Research Laboratory, Civilian American and European Surface Anthropometry Resource (CAESAR), Final Technical Report, Volume I, Summary. 